\title{
ESSAI D'UTILISATION DE L'INFRA RED MILK ANALYZER (fin)
}

\author{
par \\ J. ADDA, E. BLANC-PATIN, R. JEUNET, R. GRAPPIN, \\ G. MOCQUOT, B. POUJARDIEU, et G. RICORDEAU
}

\section{C. - Discussion concernant la partie analytique}

En ce qui concerne la reproductibilité et la précision des différentes analyses effectuées par l'appareil IRMA, les tableaux 6 et 7 résument les résultats les plus représentatifs obtenus au cours de ees essais, ainsi que ceux publiés par GOULDEN et BIGGS (1964-1967). Pour le moment, ces valeurs semblent bien correspondre aux performances que l'on peut attendre de l'appareil.

Même en considérant que la précision des 3 dosages, matière grasse, matière azotée, lactose est approximativement la même en valeur absolue, la correspondance entre IRMA et les méthodes chimiques est un peu meilleure pour la matière grasse que pour la matière azotée et un peu meilleure pour la matière azotée que pour le lactose, car le taux de matière grasse est plus variable que celui de la matière azotée, lui-même plus variable que celui du lactose. Théoriquement plus le constituant à doser possède une faible variabilité, plus la méthode d'analyse doit être fine si l'on veut que cette analyse ait une signification.

La "précision relative" de la méthode peut être estimée par le rapport, entre la variance résiduelle et la variance de l'élément à doser, qui par définition est égale à $1-\mathrm{r}^{2}$. Le tableau 6 indique que cette " présision " est de 1,6 p.100 dans le cas du dosage matière grasse, de 2,8 p.100 dans le cas du dosage de la matière azotée et de 5,7 p.100 dans le cas du dosage lactose. Avec l'IRMA la précision du dosage lactose peut paraître insuffisante puisqu'elle dépasse le seuil de 5 p. 100. Toutefois, cette précision n'est pas constante puisqu'elle est fonction de la variance de l'élément à doser dans la population considérée. D'autre part, dans de nombreux cas (paiement du lait suivant la richesse en matière grasse ou en matière azotée par exemple), c'est l'erreur absolue qui doit être prise en considération et non "l'erreur relative".

\section{Qualité et conservation des échantillons de lait}

Il est essentiel que les échantillons parvenant au laboratoire soient à la fois propres et bien conservés. 
TABLEAU VI

Précision de la méthode Irma par Rapport a UX Méthodes Chimiques

\begin{tabular}{|c|c|c|c|c|c|c|}
\hline Dosages & $\begin{array}{l}\text { Méthode } \\
\text { chimique }\end{array}$ & $n$ & $\sigma_{x y}$ & $y$ & $r^{2} x y$ & $1-r^{2} x y$ \\
\hline Matière grasse & Gerber & 305 & 0,74 & 6,15 & 0,984 & 0,016 \\
\hline Matière azotée & Kjeldahl & 71 & 0,62 & 3,56 & 0,972 & 0,028 \\
\hline Lactose ..... & Bertrand & 44 & 0,78 & 3,29 & 0,943 & 0,057 \\
\hline
\end{tabular}

$y=$ résultats IRMA ;

$y=$ écart-type de la moyenne des y en $\mathrm{g} / \mathrm{kg}$ de lait ;

$x=$ résultats méthode chimique;

$\sigma_{x y}=$ écart-type lié en $\mathrm{g} / \mathrm{kg}$ de lait;

$r^{2} x y=$ coefficient de détermination ;

$n=$ nombre d'échantillons.

TABLEAU VII

Précision et reprodudtibihité de L'APpareil Selon les aUteurs

\begin{tabular}{|c|c|c|c|c|c|c|}
\hline \multirow{2}{*}{ Auteurs } & \multicolumn{2}{|c|}{ Matière grasse } & \multicolumn{2}{|c|}{ Matière azotée } & \multicolumn{2}{|c|}{ Lactose } \\
\hline & 1 & 2 & 1 & 2 & 1 & 2 \\
\hline Goulden .......... & - & 1,02 & - & 0,68 & - & 0,71 \\
\hline Biggs $\ldots \ldots \ldots \ldots \ldots$ & 0,3 & 0,6 & 0,3 & 0,7 & 0,3 & 0,6 \\
\hline I.N.R.A. (Poligny) .... . & 0,23 & 0,74 & 0,16 & 0,62 & - & 0,78 \\
\hline
\end{tabular}

1 = écart-type des différences entre analyses faites en double exemplaire (reproductibilité).

2 = écart-type des différences entre la mesure IRMA et l'analyse effectuée par une méthode chimique de référence (précision).

a) Propreté.

Bien que l'appareil soit muni d'une série de filtres (qui donnent maintenant satisfaction), il est fortement recommandé afin d'éviter d'avoir à nettoyer trop souvent ces filtres, de ne pas analyser de laits trop sales, ces derniers n'étant malheureusement pas aussi exceptionnels qu'ils devraient l'être. En raison de la très forte absorption par l'eau la cellule de mesure n'a qu'une épaisseur interne de 40 microns et lors de nos essais nous avons très souvent été obligés pendant les premières semaines de démonter la cellule qui se trouvait bouchée malgré les filtres apparemment très fins dont était muni l'appareil. Finalement la substitution de filtres de $5 \mathrm{mi}$ crons d'ouverture à ceux de 100 microns dont était initialement pourvu l'appareil a permis d'éliminer pratiquement cet incident.

De plus, le constructeur a modifié, sur les modèles actuellement mis en vente, le profil des cellules ; aussi les phénomènes de col- 
matage par des particules étrangères au lait devraient être dans l'avenir beaucoup moins fréquents que nous ne l'avions observé au début de nos essais.

\section{b) Conservation.}

L'acidification du lait, en dehors du risque de coagulation, entraîne une transformation du lactose en acide lactique. Or l'acide lactique présente une bande d'absorption, due au groupement carboxyle, qui est très proche de celle de la matière azotée ce qui provoque une augmentation apparente de la teneur en matière azotée. Il faut donc faire en sorte que les laits soumis à l'analyse ne soit pas acides.

La lipolyse doit aussi être évitée. Elle se traduit par une diminution de la teneur en matière grasse, due à la rupture de liaisons esters, mais aussi par une augmentation apparente de la teneur en matière azotée due à la libération de molécules d'acides gras possédant une fonction carboxyle.

D'une façon générale, il faut éviter qu'il y ait une destruction des édifices moléculaires, entraînant la rupture des liaisons caractéristiques de chaque corps et l'apparition de composés susceptibles de perturber l'absorption des rayons infra-rouge aux différentes longueurs d'onde utilisées.

Il faut donc ajouter au lait un produit conservateur. Goulden (1964) préconise l'utilisation de comprimés contenant :

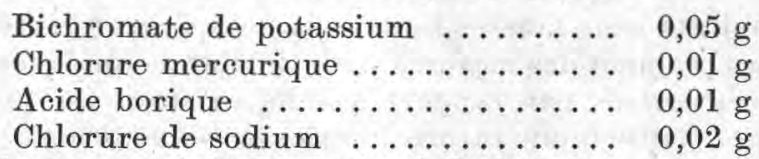

à raison de 1 comprimé pour $50 \mathrm{ml}$ de lait.

L'utilisation de bichromate de potassium n'est pas possible dans la méthode au Noir amido, car le bichromate interfère avec la lecture finale au colorimètre.

Le chlorure mercurique est habituellement employé pour la conservation des échantillons destinés au dosage de la matière azotée, par la méthode au Noir amido, et éventuellement de la matière grasse :

Le conservateur préconisé par Posthumus (1959) pour les échantillons de lait servant au dosage de la matière azotée par la méthode au Noir amido est présenté en comprimés contenant :

Chlorure mercurique .......... $0,04 \mathrm{~g}$

Fuchsine basique ............ $0,00015 \mathrm{~g}$

Chlorure de soduim ........... $0,36 \mathrm{~g}$

à raison de 1 comprimé pour $60 \mathrm{ml}$ de lait.

On peut aussi dissoudre le chlorure mercurique dans une solution hydroalcoolique à raison de $250 \mathrm{~g}$ par litre d'alcool à 95 p.100 et ajouter 5 gouttes de cette solution que l'on fait sécher dans les flacons destinés à recevoir des échantillons de lait de $60 \mathrm{ml}$. 
Il nous a donc semblé intéressant de voir si ce dernier conservateur utilisé pour le dosage de la matière azotée par le Noir amido pouvait être employé pour les échantillons de lait destinés au dosage par l'IRMA, mais les essais effectués dans ce sens ont montré que les échantillons ainsi conservés ne donnaient pas, pour les dosages effectués au moyen de l'appareil IRMA, des résultats satisfaisants notamment en ce qui concerne la détermination du taux butyreux ce qui pourrait être dû à l'activité lipolytique du chlorure mercurique déjà signalée par Manus et Bendixen (1956).

Dans l'immédiat, compte tenu de ces résultats, il semble qu'il faille écarter la formule au chlorure mercurique seul en raison de son action lipolytique et s'en tenir au conservateur préconisé par Goulden. Mais la législation française actuellement en vigueur ne permet pas la vente de comprimés contenant du chlorure mercurique. La seule solution satisfaisante consiste done à utiliser le conservateur IRMA en solution hydroalcoolique que l'on fera sécher au laboratoire dans les flacons.

\section{Organisation du laboratoire et coût de l'analyse}

\section{1. - Locaux.}

Il est souhaitable d'installer l'appareil dans une salle dont la température varie peu; une salle munie d'un dispositif de conditionnement d'air représente la solution idéale.

Les résultats sont faussés lorsque la température intérieure de l'appareil au moment des mesures varie de plus de quelques dixièmes de degré centigrade par rapport à celle existant au moment de l'étalonnage. Cette température intérieure est réglée par un thermostat, mais si l'appareil se trouve dans une pièce dont la température varie trop (en fonction de l'ensoleillement par exemple), la température intérieure de l'appareil continue à augmenter, même lorque le système de contrôle a coupé le chauffage. Nous avons expérimenté des difficultés de cet ordre aux jours les plus chauds de l'année (été 1966) et nous avons dû utiliser un ventilateur pour ramener avec plus ou moins de succès la température à la valeur désirée.

Le sol sur lequel est intallé l'appareil doit être de construction solide et à l'abri des vibrations.

Enfin il est essentiel, en dépit des dispositifs de régulation incorporés dans l'appareil, de disposer d'une alimentation électrique sans fluctuation de tension.

\section{2. - Personnel.}

Il ne sera traité ici que de la qualification du personnel, les considérations sur les effectifs seront abordées dans le paragraphe consacré aux prix de revient de l'analyse.

Il n'est pas utile que la personne chargée d'effectuer les analyses ait une qualification particulière. Il est par contre essentiel qu'il 
existe, dans chaque laboratoire utilisant l'IRMA comme appareil de routine, un technicien hautement qualifié ayant une très bonne connaissance théorique et pratique de l'appareil, capable de prévenir ou de déceler tout incident de fonctionnement et sachant intervenir dans la plupart des cas (où un incident se présente) avec rapidité et efficacité. De plus cette personne doit avoir la charge de maintenir l'étalonnage de l'appareil, opération dont dépend l'exactitude et la précision des résultats

\section{3. - Cô̂t de l'analyse.}

Toute estimation théorique en dehors d'un contexte précis repose sur un certain nombre d'hypothèses simplificatrices qui, (par leur accumulation), donnent au résultat final une marge d'incertitude assez importante Seule une expérience en vraie grandeur, permettrait de donner un prix de revient exact. On peut toutefois, en s'imposant certaines marges de sécurité, effectuer les estimations suivantes :

\section{1) Amortissement du matériel.}

Le prix actuel de l'appareil, enregistreur compris, est de 120000 F, T.T.C. En supposant que le nombre d'analyses effectuées par jour et par appareil IRMA en service ne varie pas avec la taille du laboratoire (1) la fraction du prix de revient due à l'amortissement dépend de la durée d'amortissement adoptée (tableau VIII).

Les essais réalisés à Poligny, et ceux réalisés à Guelph, montrent qu'il est possible comme l'annonce le constructeur, d'analyser 50 échantillons par heure (ce qui fournit 50 taux butyreux, 50 taux de matière azotée et 50 taux de lactose), tout en intercalant une mesure de contrôle du zéro tous les dix échantillons.

Done à raison de 50 analyses par heure, avec des journées de 8 heures et 24 jours de travail par mois, un appareil permettrait de réaliser $50 \times 8 \times 24=9600$ analyses, soit : $9600 \times 12=115200$ analyses par an. Ce chiffre reste bien entendu à vérifier car il ne repose sur aucune expérience d'utilisation de l'appareil à plein rendement sur une longue période.

On peut espérer aussi, grâce à une organisation poussée du travail, augmenter légèrement ce nombre mais, pour simplifier, les calculs seront effectués en adoptant un rythme de 110000 analyses par an.

2) Frais de personnel.

Ces frais de personnel dépendent beaucoup de l'organisation

(1) Ce qui ne correspond pas rigoureusement à la réalité, car le laboratoire qui ne possède qu'un ou deux appareils devrait, pour ne pas être débordé en cas d'incident technique, toujours travailler au-dessous de sa capacité réelle d'analyse, tandis que le grand laboratoire possédant de nombreux appareils aura toujours la possibilité de reporter sur les autres appareils les échantillons qui auraient dû être analysés par l'appareil en panne. 
TABLEAU VIII

\begin{tabular}{|c|c|c|c|c|c|}
\hline Période & 3 ans & 6 ans & 7 ans & 8 ans & 9 ans \\
\hline Valeur des annuités (en F). & 44000 & 23770 & 20780 & 18570 & 16860 \\
\hline Nombre d'analyses ....... & 110000 & 110000 & 110000 & 110000 & 110000 \\
\hline Charge par analyse (en F). & 0,40 & 0,215 & 0,19 & 0,17 & 0,155 \\
\hline
\end{tabular}

Un intérêt de 5 p. 100 a été incorporé dans l'amortissement brut.

Part de l'amortissement dans le coût de l'analyse en fonction du nombre de périodes.

des laboratoires, du coût de la main d'œuvre et de la taille du laboratoire. Deux schémas possibles seront examinés : tout d'abord celui du laboratoire classique qui utilise l'IRMA sans modifier sa structure, puis celui du laboratoire spécialisé conȩu pour l'IRMA avec son rendement maximum.

\section{Laboratoire classique.}

Il semble indispensable, quel que soit le nombre d'IRMA prévu, de recruter un technicien hautement qualifié dont l'importance a été précédemment soulignée et de lui attribuer un salaire mensuel de 2000 F justifié par la nécessité d'assurer une permanence suffisante de ce technicien dans son poste.

Il faut prévoir un opérateur par appareil mais estimons qu'il suffit d'un opérateur pour assurer le réchauffage des échantillons destinés à deux appareils ; ceei d'autant plus que l'homogénéiseur est désormais incorporé dans l'appareil.

Les résultats sont transmis sur enregistreur et peuvent facilement être transcrits par le préparateur au cours des temps morts.

Le salaire supposé attribué aux opérateurs et préparateurs est de $800 \mathrm{~F}$ (soit un salaire équivalent à celui d'un technicien 5 B dans le cadre de l'INRA). Il convient d'ajouter, les charges sociales aux sommes indiquées.

Afin de pouvoir comparer ces estimations aux prix de revient des analyses classiques, effectuées dans des laboratoires qui ont bien voulu fournir quelques informations sur leur prix de revient il est supposé que le coût du ramassage des échantillons et les frais de secrétariat ne sont pas comptés dans le prix de l'analyse mais sont supposés être inclus dans les frais généraux de l'entreprise.

La charge représentée par le personnel va en diminuant avec l'augmentation de la taille du laboratoire (tableau 9) et en additionnant les charges d'amortissement et le coût de la main d'œuvre, on arrive, sans aucun effort de compression du personnel, à un prix de revient de l'ordre de $0,50 \mathrm{~F}$, pour lequel on détermine, sur un même éehantillon l'ensemble des trois teneurs en matière grasse, matière azotée et lactose (tableau 10). 
TABLEAU IX

Evolution DU COUT DE LA MAIN-D'GUVRE EN FONCTION DE LA TAILLE DU LABORATOIRE

\begin{tabular}{c|c|c|c|c|c|c}
\hline \hline $\begin{array}{c}\text { Nombre } \\
\text { I.R.M.A. }\end{array}$ & $\begin{array}{c}\text { Techn. } \\
\text { Sup. }\end{array}$ & Opérateur & $\begin{array}{c}\text { Prépa- } \\
\text { rateur }\end{array}$ & Total & $\begin{array}{r}\text { Salaire mensuel } \\
+ \\
\text { charges sociales }\end{array}$ & $\begin{array}{c}\text { Coût par ana- } \\
\text { lyse F }\end{array}$ \\
\cline { 2 - 3 } 2 & 1 & 2 & 1 & 4 & 5720 & 0,30 \\
4 & 1 & 4 & 2 & 7 & 8920 & 0,24 \\
6 & 1 & 6 & 3 & 10 & 12120 & 0,22 \\
8 & 1 & 8 & 4 & 13 & 15320 & 0,20 \\
\hline \hline
\end{tabular}

TABLEAU $\mathrm{X}$

Prix de revient de L'analyse (amortissement + main-d'Guvre) EN FRANCS

\begin{tabular}{|c|c|c|c|c|}
\hline \multirow{2}{*}{$\begin{array}{c}\text { Années } \\
\text { d'amortissement }\end{array}$} & \multicolumn{4}{|c|}{ Nombre d'appareils } \\
\hline & 2 & 4 & 6 & 8 \\
\hline $\begin{array}{l}3 \\
5 \\
7\end{array}$ & $\begin{array}{l}0,74 \\
0,55 \\
0,49\end{array}$ & $\begin{array}{l}0,68 \\
0,49 \\
0,43\end{array}$ & $\begin{array}{l}0,66 \\
0,47 \\
0,41\end{array}$ & $\begin{array}{l}0,64 \\
0,45 \\
0,39\end{array}$ \\
\hline
\end{tabular}

Laboratoire spécialisé.

Un laboratoire spécialisé devrait être conçu de façon à minimiser les interventions manuelles. Un moyen de réaliser cette économie de main d'œuvre est d'asservir l'ensemble des IRMA à un ordinateur qui, par l'intermédiaire d'un programme, commanderait toute la séquence des opérations, enregistrerait les données et les transcrirait grâce à un lecteur perforateur de cartes.

Le rôle des manipulateurs se réduirait alors à communiquer à l'ordinateur le code identifiant l'analyse et à introduire l'échantillon dans l'appareil. C'est pourquoi il est possible de ne prévoir que 4 manipulateurs pour 8 appareils. Une telle organisation permet en outre d'éliminer les travaux d'écriture avec les erreurs qui s'y attachent et d'organiser l'analyse des échantillons de la façon la plus efficace possible, sans perdre de temps à les classer en fonction de leur origine.

L'incidence des différents postes de dépense sur le prix de l'analyse des 3 constituants d'un échantillon apparaît dans le tableau XI où le coût de l'analyse est calculé sans accorder de valeur résiduelle aux différents matériels à l'issue de la période d'amortissement. 


\section{TABLEAU XI}

INOIDENCE DES DIFFÉRENTS POSTES DE DÉPENSE SUR LE PRIX DE L'ANALYSE

Achat d'équipement (amortissable en 5 ans):

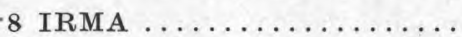

Matériel électronique $\ldots \ldots \ldots$.

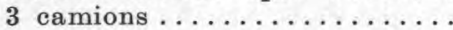

$1 \quad 175000$

5

Loyer ou amortissement d'un bâtiment de $600000 \mathrm{~F}$ en 10 ans

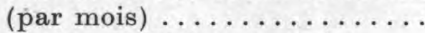
Location I.B.M. (par mois) ...

\section{Personnel :}

1 responsable Irma .........

1 chef de laboratoire ........

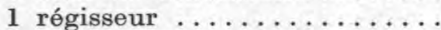

4 manipulateurs Irma ......

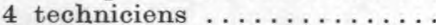

3 chauffeurs ..............

1 secrétaire .............

2 femmes de ménage .......

1 aide-chimiste.......... 18

Charges sociales $25 \% \quad \ldots \ldots$

Frais de fonctionnement : Electricité, verrerie et divers.. Transport ............

$$
\begin{array}{rlll}
960 & 000 & \mathrm{~F} \\
125 & 000 & - \\
90 & 000 & - \\
175 & 000 & \mathrm{~F}
\end{array}
$$

$=235000 \mathrm{~F}$

Soit par analyse . 0,27 F \begin{tabular}{lll}
6 & 000 & $\mathrm{~F}$ \\
8 & 000 & - \\
\hline 14 & 000 & $\mathrm{~F}$
\end{tabular}

Soit par analyse. $0,18 \mathrm{~F}$

$$
\begin{array}{rlr}
2 & 000 & \mathrm{~F} \\
2 & 000 & - \\
2 & 000 & - \\
3 & 200 & - \\
3 & 200 & - \\
2 & 400 & - \\
& 800 & - \\
1 & 200 & - \\
& 800 & - \\
\hline 17 & 600 & \mathrm{~F} \\
4 & 400 & - \\
\hline 22 & 000 & \mathrm{~F}
\end{array}
$$

Soit par analyse. $0,28 \mathrm{~F}$

$40000 \mathrm{~F}$ 60000

\begin{tabular}{l} 
Soit par analyse . $\frac{0,11 \mathrm{~F}}{0,84 \mathrm{~F}}$ \\
Total $\ldots \ldots \ldots \ldots$ \\
\hline
\end{tabular}

Ce prix de revient, pour hypothétique qu'il soit à l'heure actuelle, peut être considéré comme étant du même ordre de grandeur que ceux obtenus dans les laboratoires utilisant les méthodes d'analyses chimiques classiques, Gerber et Noir amido.

\section{Discussion}

Il semble qu'un certain nombre de critiques formulées dans les pages précédentes vis-à-vis de l'appareil IRMA sont dépassées, au moment où nous terminons la rédaction de ce texte, puisque certains éléments de l'appareil : homogénéisation, système optique, 
réglage de la température, ect... seront, aux dires du constructeur, modifiées sur la deuxième version (MK II) de l'appareil.

Avant de porter un jugement définitif sur les performances de l'appareil, il faudrait, après le premier stade expérimental que nous venons de décrire, travailler pendant une période de temps assez longue dans les conditions proches des conditions réelles d'utilisation.

Toutefois il apparait déjà que, par ses qualités (rapidité, absence de réactif acide, possibilité d'enregistrement mécanographique et prix de revient de l'analyse acceptable), l'Infra Red Milk Analyzer est un appareil qui répond exactement aux conditions requises pour faire des analyses de lait en série.

Son utilisation se justifiera donc chaque fois qu'il faudra faire des dosages de matière grasse, de matière azotée, et éventuellement de lactose, sur un nombre élevé d'échantillons de lait. C'est essentiellement ce que montre cette étude. Cependant, compte tenu de la rapidité avec laquelle il est possible d'obtenir la composition en matière grasse et en matière azotée d'un échantillon de lait il semble que cet appareil puisse aussi être utile pour standardiser les laits de fabrication en fromagerie. En effet le réglage du rapport matière azotée/matière grasse, du lait est important pour le transformateur, car, de la précision de l'ajustement du taux butyreux du lait de fabrieation en fonction de son taux de matière azotée dépend en grande partie la composition du fromage et en particulier sa teneur en matière grasse.

Des essais en laboratoire ont montré qu'il est possible de régler avec une précision acceptable le rapport matière azotée/matière grasse du lait en un temps ne dépassant pas 3 minutes si l'on ne tient compte que des opérations essentielles qui sont les suivantes: dosage de la matiére grasse et de la matière azotée du lait de mélange, lecture sur un abaque de la quantité de lait écrémé à ajouter, vérification de l'exactitude du rapport, matière azotée/matière grasse, obtenu.

Mais le temps requis pour l'ensemble des opérations devient notablement plus long, s'il est nécessaire de réchauffer les échantillons à $40^{\circ} \mathrm{C}$ avant de les homogénéiser. Un dispositif permettant un réchauffage presque instantané du lait devrait donc être imaginé pour que le réglage du rapport matière azotée/matière grasse soit réalisé en un temps compatible avec les exigences de la fabrication. Il en reste en outre à démontrer que l'utilisation de l'appareil dans le contexte d'un laboratoire d'usine est possible.

L'Infra Red Milk Analyzer présente des qualités incontestables mais son utilisation doit être envisagée avec une grande prudence car c'est un appareil complexe qu'il convient de faire utiliser seulement par un personnel compétent sous peine de courir au devant des plus graves déboires. En dehors de toute considération de rentabilité économique il ne paraît pas souhaitable, et ceci dans leur 
propre intérêt, que de nombreux petits laboratoires s'équipent avec l'IRMA. On peut estimer, à la rigueur, qu'ils pourraient le faire sans trop de risques sous la tutelle d'un laboratoire central interprofessionnel qui les aiderait à traverser la difficile période d'adaptation. Mais, à nos yeux, la solution de choix consiste à créer des laboratoires spécialisés, résultant d'un regroupement dont l'importance justifierait l'acquisition de 4 à 5 appareils au moins et leur permettrait ainsi de fonctionner dans les meilleures conditions.

\section{Résumé}

L'Infra Red Milk Analyzer a été utilisé pour le dosage de la matière grasse, de la matière azotée, et du lactose dans le lait. La reproductibilité est de $0,23 \mathrm{~g} / \mathrm{kg}$ pour la matière grasse et de $0,16 \mathrm{~g} / \mathrm{kg}$ pour la matière azotée.

La précision des résultats, par rapport aux méthodes chimiques est de $0,74 \mathrm{~g} / \mathrm{kg}$ pour la matière grasse, $0,62 \mathrm{~g} / \mathrm{kg}$ pour la matière azotée, et $0,78 \mathrm{~g} / \mathrm{kg}$ pour le lactose.

Des calculs de prix de revient montrent qu'il est possible de réaliser l'analyse des 3 composants pour un prix de revient du même ordre de grandeur que celui obtenu dans les laboratoire. utilisant les méthodes d'analyses chimiques (pour la graisse et les matières azotées seulement).

\section{Summary}

The Infra-Red-Milk-Analyzer (IRMA) has been used for fat, protein and lactose determinations in milk. Reproducibility was found to be $0,23 \mathrm{~g} / \mathrm{kg}$ for fat and $0,16 \mathrm{~g} / \mathrm{kg}$ for protein.

When compared with chemical methods, the accuracy of IRMA was $0,74 \mathrm{~g} / \mathrm{kg}$ for fat, $0,62 \mathrm{~g} / \mathrm{kg}$ for protein and $0,78 \mathrm{~g} / \mathrm{kg}$ for lactose.

The eosts of milk analysis by IRMA (for both fat, protein and lactose) is of the same order of magnitude as the cost of routine analysis in laboratories using classical chemical methods (for fat and protein only).

\section{RÉFÉRENCES}

Alais C., 1965. Rev. Lait. Fr., 227, 624.

Biggs D. A., 1967. J. Dairy Sci., 50, 5, 803.

GOULDEN J. D. S., 1964. J. Dairy Res., 31, 273.

Goulden J. D. S., Shtelds J. et Haswell R., 1964. J. Soc. Dairy Techn., 17, 1. Manus L. N. et Bendixen H. A., 1956. J. Dairy Sci., 39, 508.

Posтнuмus S., 1959. Landbouwvoorlichting, 69, 16.

Rowland S. J., 1938. J. Dairy Res., 9, 42.

Terroine E., 1951. Ann. Nutr. et Aliment., 5, 6, 538. 